The resources required for research and education related to cholesterol reduction include many in which general internists can already claim a track record: health promotion and disease prevention, behavioral medicine, computers in medicine, medical education, clinical epidemiology, health services research, decision analysis, and medical economics, to name a few. General internal medicine has demonstrated the ability to integrate these disciplines. The development and application of knowledge related to the prevention of coronary heart disease through lowering blood cholesterol should provide a major new patientcare challenge for general internal medicine - and what an opportunity! - David S. Siscovick, MD, Associate Editor

\section{REFERENCES}

1. Report of Inter-Society Commission for Heart Disease Resources: Optimal resources for primary prevention of atherosclerotic diseases. Circulation 1984:70:155A-205A

2. The Lipid Research Clinics Coronary Primary Prevention Trial results. I, Reduction in incidence of coronary heart disease. Lipid Research Clinics Program. Lipid Metabolism-Atherogenesis Branch, National Heart. Lung. and Blood Institute. JAMA 1984:251:351

3. The Lipid Research Clinics Coronary Primary Prevention Trial results. II. The relationship of reduction in incidence of coronary heart disease to cholesterol lowering. Lipid Research Clinics Program. Lipid Metabolism-Atherogenesis Branch, National Heart, Lung, and Blood Institute. JAMA 1984:251:356

4. National Institute of Health Consensus Development Conference Statement: Lowering blood cholesterol to prevent heart disease. JAMA 1985:253:14,2080-2086

5. Peters WL, Gorroll AH. New approaches to the evaluation and treatment of hypercholesterolemia in primary care practice. J Gen Intern Med 1986:1:183-95

\title{
Studying and Teaching the Gatekeeper
}

THE GATEKEEPER concept uses case management as one proposed solution to the problem of rising medical care costs. The plan places the primary care physician squarely at the entrance to the medical care system. Primary care physicians control the care that their patients get, unless the patients are willing to pay more out-of-pocket. Without the casemanaging physician's approval, the plan will not pay for medical care. In most gatekeeper plans, the primary care physicion has an incentive to save money by ordering fewer services.

Case management is being adopted by increasing numbers of prepaid groups and individual practice associations, and it is now being considered by Medicaid plans around the nation. Although the gatekeeper plan often is thought of as a component of prepaid practice, neither is dependent on the other. Not all prepaid plans use the strict gatekeeper system, and one could imagine $\alpha$ fee-for-service system that would not pay for services unless they had been approved by a gatekeeping physicion.

In the gatekeeper plan, primary care physicians have the mandate to serve as their patients' advocates, as their guides through the increasingly specialized medical maze, but also as the rationer of their medical services. It is the task of rationing that makes the gatekeeper concept unlike traditional delivery systems. In the role of rationer, the physician must become guardian for the medical purse as well as the patient's health. Now we are being asked to allocate health resources and health dollars closer to the level of care - not in the board rooms of hospitals, in the halls of Congress or in the offices of the Health Care Financing Adminis- tration, but in the physician's exam room and at the bedside.

The emergence of this concept of physician as rationer issues a mandate for researchers and educators in general internal medicine and other primary care disciplines to answer some of the fundamental questions posed by case management and to find better ways of preparing future physicians to fulfill this role.

\section{RESEARCH ISSUES}

It would seem self-evident that gatekeeper plans should be adopted on a widespread basis only after critical evaluation of their impact and demonstration of their effectiveness. Although experiments with gatekeeper plans are now being implemented, there is really no solid evidence that they save money or provide superior medical care. Nor is there satisfactory evidence that the quality or access to care suffers. In fact, the current "experiments" are actually "demonstration projects," since few of the gatekeeper plans are designed with control groups in a rigorous experimental design. Whatever we call these ventures, most of them have little or no critical evaluation, including the mandatory Medicaid plans.

Will gatekeeper plans be health care delivery's version of gastric freezing - highly touted but ineffective, even harmful? These systems could have adverse effects on the access to care and its quality because of the incentives that may encourage underutilization and denial of care. On the other hand, access and quality of care could be improved because of more continuous and coordinated care. 
How will we identify the more subtle but important outcomes? Evaluation will require that proper yardsticks be used for measurement, including new and valid indicators of clinical outcomes, functional status, access and patient satisfaction.

Clinical research will be as important in the gatekeeper arrangement as it is in traditional medical care, but the emphasis on primary care highlights the need for data relevant to ambulatory practice. Knowing which medical interventions yield the most quality in diagnosis and mangement for the least cost is crucial to the gatekeeper concept. Hopefully, we will be able to turn to publications such as the Journal OF GENERAL INTERNAL MEDICINE for these data, but today most searches for good cost-effectiveness data are dry taps. It is in these uncertain clinical situations that wide variations seem to exist in medical practice, and nonclinical influences on decision making (such as the physician's economic benefit) probably play influential roles.

The gatekeeper concept is likely to put stress on the physician-patient relationship because it creates ethical as well as economic risks. The physician and the patient may even become adversaries. This compels us to investigate how the ethical dilemma of physicians acting simultaneously as agents of their patients and advocates of cost containment can be addressed. We need to understand better how our values and preferences differ from those of our patients.

When funding for research and teaching in health services research seems scarcer than ever, we have a special opportunity for research in a new laboratory of medical care. Will the Federal government, which is encouraging Medicare beneficiaries to participate in prepaid plans and approving Medicaid capitation programs, will states that are sponsoring the Medicaid gatekeeper plans, and will organizations that are implementing them spend even a small fraction of their funds on impartial, well-designed evaluations? Each of these parties would benefit from the knowledge gained from careful evaluations of case management.

\section{EDUCATIONAL ISSUES}

In addition to taking on the research questions raised by case management, general internal medicine programs con assume leadership in training and preparing future physicians for the gatekeeper role. Screening and prevention technology, clinical epidemiology, clinical decision making, clinical economics, and clinical ethics are critical applied basic sciences for medical practice, and can help physicians fulfill the difficult responsibilities of the gatekeeper. The challenge is to integrate these sci- ences into teaching programs for housestaff and students.

The organization of this education needs to be examined. Residents and students need to spend time in ambulatory settings, but saying only that is insufficient. Ambulatory care training in subspecialty as well as primary care is important, since future gatekeepers should learn to recognize common disorders, weigh treatment options, and identify when referral is necessary. Federal or residency review committee regulations that mandate upper or lower limits to different types of ambulatory care interfere with the ability of program directors to be innovative, given the special and sometimes unique opportunities available at different training programs.

If general internal medicine programs are to assume leadership in training physicians for the gatekeeper role, should they now seek to diversify their practices by becoming directly involved in these systems? Certainly there are advantages. One of the most exciting challenges in gatekeeper plans is that they place the primary care physician in charge of the care of $\alpha$ defined population. Can we educate internists to think of their patients in the context of a population? The relationship between epidemiology and clinical practice becomes real when the doctor has to practice denominator-based medicine. The gatekeeper plan could help to overcome epidemiologic nihilism in clinical education.

Although there are potential advantages for primary care educators who teach in gatekeeper systems, the sword is double-edged. Most residencies have needed hospital subsidies for their teaching clinics in the era of conventional fee-for-service payment. If gatekeeper plans pay a standard capitation rate to teaching practices that already care for some of the most expensive primary care patients, and admit them to some of the nation's most expensive hospitals, there will be even fewer resources left to pay for education. While the gatekeeper plan puts the primary care physician in the driver's seat, there may not be enough gas in the tank for primary care education.

The gatekeeper model is, in a sense, a new technology - $\alpha$ new method of delivering medical care - and it presents new challenges for research and education in primary care internal medicine. It deserves at least as much attention as a new drug or another new technology would receive. The gatekeeper plan's efficacy should be proven and its side-effects identified before it is adopted on a widespread basis - Neil R. Powe, MD, $M P H$, and John M. Eisenberg, MD, MBA, Section of General Medicine, Leonard Davis Institute of Health Economics and Robert Wood Johnson Foundation Clinical Scholars Program, University of Pennsylvania, Philadelphia, Pennsylvania. 